\title{
Upregulation of microRNA-24 causes vasospasm following subarachnoid hemorrhage by suppressing the expression of endothelial nitric oxide synthase
}

\author{
HUAN-TING LI ${ }^{1}$, JING WANG ${ }^{2}$, SHI-FANG LI ${ }^{1}$, LEI CHENG ${ }^{1}$, WAN-ZHONG TANG ${ }^{1}$ and YU-GONG FENG ${ }^{1}$ \\ Departments of ${ }^{1}$ Neurosurgery and ${ }^{2}$ Oncology, The Affiliated Hospital of Qingdao University, \\ Qingdao, Shandong 266003, P.R. China
}

Received January 24, 2016; Accepted January 12, 2017

DOI: $10.3892 / \mathrm{mmr} .2018 .9050$

\begin{abstract}
MicroRNA (miR)-24 has been reported to associate with various diseases by acting on different signaling pathways. The present study aimed to elucidate the association between miR-24 expression levels and vasospasm following subarachnoid hemorrhage (SAH), and its underlying mechanism. An miR online database was searched, identifying endothelial nitric oxide synthase (NOS3) as a potential target gene of miR-24. A luciferase reporter assay performed to investigate the regulatory association between miR-24 and NOS3 revealed that miR-24 bound to the NOS3 3 ' untranslated region and inhibited NOS3 expression. Reverse transcription-quantitative polymerase chain reaction and western blot analysis were performed to investigate the miR-24 and NOS3 expression levels in samples from patients with $\mathrm{SAH}$, and demonstrated a negative correlation between the two. In addition, miR-24 expression levels were increased in SAH patients with vasospasm compared with those without, whereas the opposite results were observed for NOS3. Vascular smooth muscle cells (VSMCs) transfected with an miR-24 inhibitor exhibited increased expression levels of NOS3, whereas those transfected with an miR-24 mimic or NOS3 small interfering RNA exhibited reduced expression levels of NOS3, compared with the control. These results indicated a negative regulatory association between miR-24 and NOS3. Downregulation of NOS3 may induce vasospasm following $\mathrm{SAH}$, which may be due to the upregualtion of miR-24 in VSMCs.
\end{abstract}

Correspondence to: Dr Yu-Gong Feng, Department of Neurosurgery, The Affiliated Hospital of Qingdao University, 16 Jiangsu Road, Qingdao, Shandong 266003, P.R. China E-mail: depressionnos1@163.com

Key words: microRNA-24, vasospasm, subarachnoid hemorrhage, endothelial nitric oxide synthase

\section{Introduction}

Aneurysmal subarachnoid hemorrhage (SAH) is a severe and life-threatening disease, which results in fatality in $27-44 \%$ of patients; $46 \%$ of survivors live with severe functional and cognitive impairment $(1,2)$. The response to $\mathrm{SAH}$ involves securing the cerebral aneurysms and treating the cerebral vasospasm, which occurs in $70 \%$ of SAH patients between 3 and 14 days following SAH (3). Cerebral vasospasm results in delayed cerebral ischemia and is primarily responsible for unfavorable results and even mortality in SAH patients (1). However, currently no definitive therapy is available to address this complication.

The endothelial nitric oxide synthase (NOS3) gene in vessel endothelium continuously produces nitric oxide (NO) to maintain basal vascular tone (4). As a result of aneurysmal SAH, the expression of the NOS3 gene is altered and the balanced modulation of cerebral vascular tone is interrupted $(5,6)$. Previous studies have reported aberrant cerebrospinal fluid NO levels in humans following SAH $(7,8)$. In addition, adenovirus gene transfer of NOS3 in dogs and in ex vivo human arteries has demonstrated protective effects in $\mathrm{SAH}(6,9)$. NO serves a role in the inhibition of inflammation and proliferation of smooth muscle, pathological alterations which are observed in cerebral vasospasm $(10,11)$. Previous studies have suggested a correlation between NOS3 polymorphisms and the development of intracranial aneurysms as well as coronary spasm, which has physiological similarity to cerebral vasospasm $(12,13)$.

MicroRNAs (miRNAs) are non-coding RNAs consisting of 22 nucleotides. They act as meta-regulators of gene expression and are pivotal for cellular alterations that are required for the process of development. miRNAs in the brain serve important roles in the formation and function of the dendritic spine, and in the synaptic plasticity that is necessary for normal cognitive function. A thorough understanding of the mechanisms underlying the regulation of miRNA expression is important, as abnormal regulation of miRNAs has been associated with an array of neurological disorders (14).

miRNAs additionally serve as regulators of vascular phenotype via suppression or maintenance of differentiation (15). In SAH, the identification of blood borne biomarkers is required to estimate the risk of late cerebral 
ischemia (LCI) and cerebral vasospasm. Previous studies have demonstrated numerous alterations in gene expression in cerebral arteries in response to various types of strokes including focal cerebral ischemia and $\mathrm{SAH}$, and following cardiac arrest (16-18). It has been demonstrated that stimulation of certain signal transduction pathways in the cerebral vasculature following stroke results in transcriptional regulation of proteins, inflammatory mediators and vasoconstrictor receptors engaged in the maintenance of the integrity of the blood-brain barrier (19). However, regulation may occur at other levels, and SAH may cause alterations in miRNA expression in cerebral arteries, which following release into serum may as act as biomarkers to estimate the risk of LCI and cerebral vasospasm.

Differential expression of miRNA (miR)-24 has been identified in vascular tissue from SAH patients with vasospasm (20), and dysregulation of NOS3 has been revealed to be involved in the underlying molecular mechanism of vasospasm following SAH $(21,22)$. Using an online miRNA database, NOS3 was identified as a potential target of miR-24. The present study validated NOS3 as a target gene of miR-24 and investigated the involvement of miR-24 and NOS3 in the development of vasospasm following SAH.

\section{Materials and methods}

Sample collection. The present study was approved by the Human Ethics Committee of Qingdao University (Qingdao, China). A total of $29 \mathrm{SAH}$ patients with $(n=13)$ or without $(n=16)$ vasospasm (age, 21-75) were recruited from the Department of Neurosurgery, The Affiliated Hospital of Qingdao University (Qingdao, China) between December 2013 and December 2014. Diagnosis was performed by cerebral angiogram, computed tomography scan (Fisher grade $\geq 2$ ) and computed tomographic angiogram. Participants or their first-degree relatives signed informed consent forms prior to samples being obtained. Patients underwent surgical clipping, endovascular coiling or a combination of the two. The blood samples were obtained on day 7 after the onset of SAH. Leukocytes were isolated from the blood using Human Peripheral Blood Leukocytes Isolation kit (Beijing Solarbio Science \& Technology Co., Ltd., Beijing, China) followed by centrifugation for $1 \mathrm{~h}$ at $700 \mathrm{xg}$ and $37^{\circ} \mathrm{C}$. The isolated leukocytes were subsequently used for RNA extraction and functional experiments.

Cell culture and transfection. Vascular smooth muscle cells (VSMCs; American Type Culture Collection, Manassas, VA, USA) were cultured in Dulbecco's modified Eagle's medium (DMEM; Invitrogen; Thermo Fisher Scientific, Inc., Waltham, MA, USA), supplemented with $1 \%$ penicillin/streptomycin, $2 \mathrm{mM}$ glutamine and $10 \%$ fetal bovine serum (FBS; Invitrogen; Thermo Fisher Scientific, Inc.), at $37^{\circ} \mathrm{C}$ in $5 \% \mathrm{CO}_{2}$. A total of $12 \mathrm{~h}$ later, VSMCs in DMEM (Invitrogen; Thermo Fisher Scientific, Inc.) without antibiotics were seeded into 6-well plates at a density of $3-6 \times 10^{5}$ cells/well. A total of $24 \mathrm{~h}$ following this, at a confluence of $80 \%$, Lipofectamine ${ }^{\circledR} 2000$ (Invitrogen; Thermo Fisher Scientific, Inc.) was used to transfect: i) miR-24 mimic (UGGCUCAGUUCAGCAGGAACAG; cat. no. AM17100; Ambion; Thermo Fisher Scientific, Inc.); ii) miR-24 inhibitor oligonucleotide (GAGCUUCCAGGUAGCAGGUAGCAG
GGCUGCUGUUCUGAGCUGUGGAUUGGACCCGCCCU CCGGUGCCUACUGAGCUGAUAACAGUUCUGAUUUUA CACACUGGCUCAGUUCAGCAGGAACAGGAGUCGAGC CCGAGAGCAAAAAAGACUA; cat. no. AM17000; Ambion, Thermo Fisher Scientific, Inc.); iii) scrambled oligonucleotide (ACUGUUCUGAUUUUACACACUGGCUC; $\mathrm{Cy} 3$ dye-labeled anti-miR negative control; cat. no. AM17011; Ambion; Thermo Fisher Scientific, Inc.); and iv) NOS3 siRNA (forward, 5'-GAA GAGGAAGGAGUCCAGUAACACA-3' and reverse, 5'-UGU GUUACUGGACUCCUUCCUCUUC-3'; cat. no. AM17000; Ambion; Thermo Fisher Scientific, Inc.) into VSMCs according to the manufacturer's protocol. In brief, cells at $\sim 50 \%$ confluence on an 100-mm culture dish were treated with a mixture of $30 \mathrm{nM}$ siRNA and $1.25 \mu \mathrm{l} / \mathrm{ml}$ Lipofectamine ${ }^{\mathrm{TM}}$ RNAiMAX (Thermo Fisher Scientific, Inc.) in $5 \mathrm{ml}$ of Opti-MEM (Gibco; Thermo Fisher Scientific, Inc.) for $3 \mathrm{~h}$ at $37^{\circ} \mathrm{C}$.

$R N A$ isolation and reverse transcription-quantitative polymerase chain reaction ( $R T-q P C R)$. To analyze the expression levels of NOS3 mRNA and miR-24, a NucleoSpin miRNA kit (Machery-Nagel GmbH, Düren, Germany) was used to isolate total RNA from VSMCs or patient samples according to the manufacturer's protocol. The Agilent RNA 6000 Nano kit and a 2100 Bioanalyzer (Agilent Technologies, Inc., Santa Clara, CA, USA) were used to determine the integrity of the total RNA and a NanoDrop 2000c (Thermo Fisher Scientific, Inc.) was used to measure RNA concentration. A Universal cDNA Synthesis kit (Machery-Nagel $\mathrm{GmbH}$ ) was used to reverse-transcribe RNA to cDNA according to the manufacturer's protocol. A SYBR ${ }^{\circledR}$ Green Master mix (Thermo Fisher Scientific, Inc.) was used to perform qPCR analysis of NOS3 mRNA expression, using $4 \mu 1$ diluted cDNA in a final reaction volume of $10 \mu \mathrm{l}$. The thermocycling conditions were as follows: $20 \mathrm{sec}$ at $95^{\circ} \mathrm{C}, 3 \mathrm{sec}$ at $95^{\circ} \mathrm{C}$, followed by 40 cycles of $3 \mathrm{sec}$ at $95^{\circ} \mathrm{C}$ and $30 \mathrm{sec}$ at $60^{\circ} \mathrm{C}$. A TaqMan ${ }^{\circledR}$ miRNA assay (Applied Biosystems; Thermo Fisher Scientific, Inc.) was used to detect the expression of miR-24. The following thermocycling conditions were used: $5 \mathrm{~min}$ at $95^{\circ} \mathrm{C}$; followed by 45 cycles of $5 \mathrm{sec}$ at $95^{\circ} \mathrm{C}, 5 \mathrm{sec}$ at $60^{\circ} \mathrm{C}$ and $10 \mathrm{sec}$ at $72^{\circ} \mathrm{C}$. The $2^{-\Delta \Delta \mathrm{Cq}}$ method was used to normalize the data (23). U6 was used for normalization of expression of miR-24 and GAPDH for NOS3. Each experiment was performed in triplicate. The sequences of PCR primers were: NOS3 (forward: 5'-TGC TGGCATACAGGACTCAG-3'; reverse: 5'-TAGGTCTTGGG GTTGTCAGG-3'); miR-24 (forward: 5'-ACACTCCAGCTG GGTGGCTCAGTTCAGCAG-3'; reverse: 5'-CTCAACTGG TGTCGTGGAGTCGGCAATTCAG-3'); GAPDH (forward: 5'-GCCAAAAGGGTCATC ATCTC-3'; reverse: 5'-GTAGAG GCAGGGATGATGTTC-3'; U6 (forward: 5'-CTCGCTTCG GCAGCACA-3', reverse: 5'-AACGCTTCACGAATTTGC GT-3').

Luciferase assay. To confirm whether NOS3 expression was directly regulated by miR-24, the 3 ' untranslated region(UTR) of NOS3 was amplified by PCR, and the PCR product was inserted into a pLUC reporter vector (Promega Corporation, Madison, WI, USA). As a control, a mutant 3'UTR was inserted. The sequences of the wild-type and mutant NOS3 3'UTRs are presented in Fig. 1. Lipofectamine 2000 was used to co-transfect the VSMCs with miR-24 at a final concentration 
of $100 \mathrm{nM} /$ well, using $0.4 \mathrm{mg}$ firefly luciferase reporter vector containing the 3'UTR sequences and $0.02 \mathrm{mg}$ control pRL-CMV plasmid containing Renilla luciferase (Promega Corporation). Cells were cultured for $48 \mathrm{~h}$, following which the Dual-Luciferase Reporter assay system (Promega Corporation) was used to assess the luciferase activity according to the manufacturer's protocol. Renilla luciferase activity was used to normalize the values. Each experiment was performed at least 3 times.

Western blot analysis. VSMCs or patient samples were lysed with radioimmunoprecipitation assay buffer (Sigma-Aldrich; Merck Millipore, Darmstadt, Germany) containing protease inhibitors (Roche Applied Science, Madison, WI, USA). Lysates were obtained by centrifugation for $15 \mathrm{~min}$ $12,000 \times \mathrm{g}$ at $4^{\circ} \mathrm{C}$. A Bio-Rad Protein microassay (Bio-Rad Laboratories, Inc., Hercules, CA, USA) was performed to estimate the protein concentration, and boiling water was used to heat the samples. A $12 \%$ SDS-PAGE was used to separate the target protein (35 $\mu \mathrm{g} /$ lane) according to manufacturer's protocol. The proteins were transferred from the gel to a polyvinylidene difluoride (PVDF) membrane (PerkinElmer, Inc., Waltham, MA, USA) by electroblotting for $2 \mathrm{~h}$ at $90 \mathrm{~V}$. The membrane was incubated with $5 \%$ non-fat milk powder in TBS containing $0.1 \%$ Tween 20 in the dark at $4^{\circ} \mathrm{C}$ for $2 \mathrm{~h}$ to prevent nonspecific binding. Membranes were subsequently incubated with a mouse anti-human NOS3 antibody (1:1,000; cat. no. sc-376751; Santa Cruz Biotechnology, Inc., Dallas, TX, USA) or a mouse anti-human $\beta$-actin antibody (1:1,000; cat. no. sc-418965; Santa Cruz Biotechnology, Inc.) in $5 \%$ non-fat milk for $16 \mathrm{~h}$ at $4{ }^{\circ} \mathrm{C}$, following which membranes were washed twice with PBS. PVDF membranes were incubated with a rabbit anti-mouse horseradish peroxidase-conjugated secondary antibody (1:3,000; sc-2364, Cell Signaling Technology, Inc., Danvers, MA, USA) for $2 \mathrm{~h}$ at room temperature. Visualization of the protein bands was performed by incubating the membranes with an Enhanced Chemiluminescence reagent (Thermo Fisher Scientific, Inc.) for 2 min. Analysis Software (VisionWorks ${ }^{\circledR}$ LS, 97-0186-02 Single; UVP, LLC, Phoenix, AZ, USA) was used to analyze the intensity of the protein bands, and ImageJ software (National Institutes of Health, Bethesda, MD, USA) was used to determine the optical density. Three independent experiments were performed.

Statistical analysis. SPSS software version 10.0 (SPSS, Inc., Chicago, IL, USA) was used to perform statistical analyses. One-way analysis of variance was used to compare multiple groups. Data are presented as the mean \pm standard deviation of at least three independent experiments. $\mathrm{P}<0.05$ was considered to indicate a statistically significant difference.

\section{Results}

NOS3 is a predicted target gene of $m i R-24$. miR-24 has been reported to be associated with a variety of diseases by acting on different signaling pathways (24). The present study aimed to determine the association between $\mathrm{miR}-24$ expression levels in vasospasm following SAH and its underlying mechanism. It has previously been reported that miR-24 is downregulated
hsa-miR-24

Wild-type NOS3

5'-CUGCGAUUAUAGACGUGAGCCAC-3'

\|\|$\|$

hsa-miR-24

Mutant NOS3
5'-CUGCGAUUAUAGACGUGAGCCAC-3'

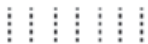

3'-GACAAGGACGACUUGGUGAUAG-5'

Figure 1. NOS3 is a predicted target gene of miR-24. The sequences of miR-24 and the NOS3 3'UTR are presented, revealing the 'seed sequence'. The potential binding site was mutated using site-directed mutagenesis to create the mutant NOS3 3'UTR sequence. miR, microRNA; NOS3, endothelial nitric oxide synthase; UTR, untranslated region.

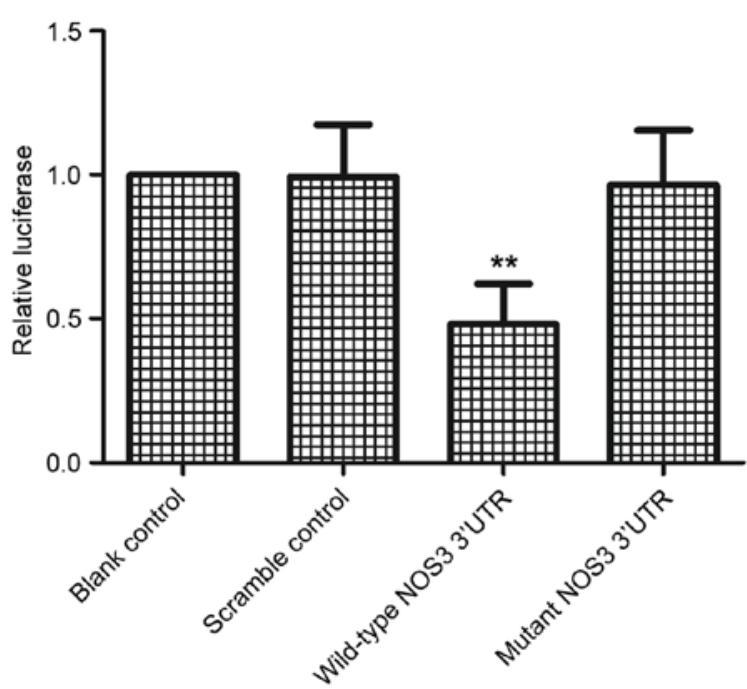

Figure 2. NOS3 is a direct target of miR-24 in VSMCs. A luciferase assay was performed in which wild-type or mutant NOS3 3'UTR were co-transfected with an miR-24 mimic or scramble control. Luciferase activity was measured, and this activity was reduced in VSMCs co-transfected with wild-type NOS3 3'UTR and miR-24, verifying NOS3 as a direct target gene of miR-24. Data are expressed as the mean \pm standard deviation. ${ }^{* *} \mathrm{P}<0.01$ vs. scramble control. miR, microRNA; NOS3, endothelial nitric oxide synthase; VSMCs, vascular smooth muscle cells; UTR, untranslated region.

in the vasculature of SAH patients with vasospasm (20). Computational analysis was performed using an online miRNA database (www.mirdb.org) to identify the possible target gene of miR-24, and NOS3 was predicted as a target of miR-24, with a potential binding site in the 3'UTR of NOS3 and was selected for further analysis based on its-physiological and pathological functions (Fig. 1).

NOS3 is a direct target of miR-24 in VSMCs. To confirm whether miR-24 and NOS3 interact, a vector containing the 3'UTR of NOS3 was constructed and a luciferase assay was performed. Only the luciferase activity from the cells co-transfected with miR-24 and wild-type NOS3 3'UTR was significantly reduced compared with the scramble control $(\mathrm{P}<0.01$; Fig. 2). The observation that mutations in the predicted binding site abolished the inhibitory effect of miR-24 on luciferase activity indicated that the predicted binding site is the real 'seed sequence' in the 3'UTR of NOS3. Therefore, the luciferase assay suggested that miR-24 binds to the NOS3 3'UTR, resulting in a significant decrease in luciferase activity compared with the scramble control. 


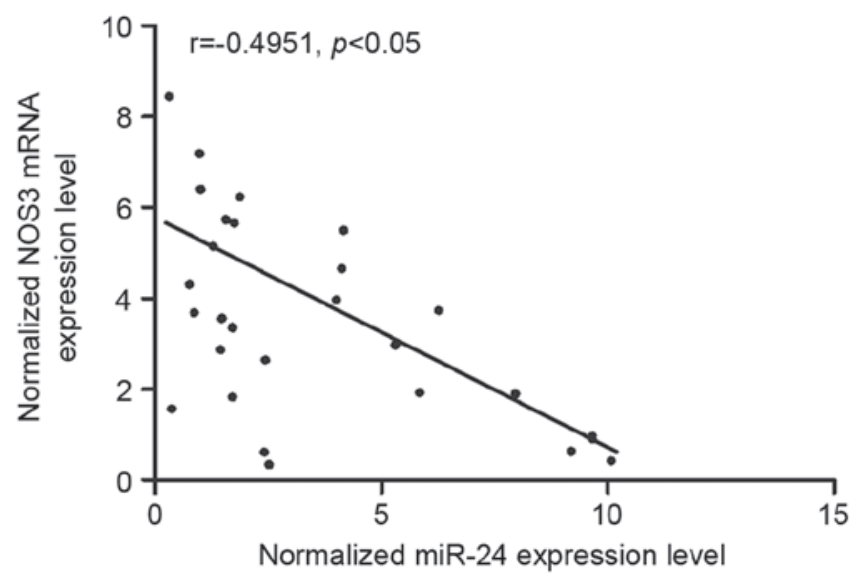

Figure 3. Negative regulatory association between miR-24 and NOS3. miR-24 and NOS3 expression levels were analyzed by reverse transcription-quantitative polymerase chain reaction in vascular tissues from 29 subarachnoid hemorrhage patients. There was a negative correlation between miR-24 and NOS3 expression levels. $\mathrm{P}<0.05$. miR, microRNA; NOS3, endothelial nitric oxide synthase.

Negative regulatory association between miR-24 and NOS3. Vascular tissues were collected from SAH patients with $(n=13)$ or without $(n-16)$ vasospasm and used used to investigate the interaction between miR-24 and NOS3. Analysis of the expression levels of miR-24 and NOS3 mRNA in the 29 tissues demonstrated that the two were negatively correlated (Fig. 3), confirming the negative regulatory association between miR-24 and NOS3.

Comparison of expression levels of miR-24 and NOS3 in SAH patients with or without vasospasm. RT-qPCR and western blot analysis were performed to detect and compare the expression levels of miR-24 and the mRNA and protein expression levels of NOS3 between vascular tissues from SAH patients with or without vasospasm. miR-24 expression levels were increased in SAH patients with vasospasm (Fig. 4A) compared with those without vasospasm $(\mathrm{P}<0.01)$, whereas the mRNA (Fig. 4B) and protein (Fig. 4C) expression levels of NOS3 were decreased in $\mathrm{SAH}$ patients with vasospasm compared with those without vasospasm $(\mathrm{P}<0.01)$. These findings indicated that downregualtion of NOS3 caused by the upregulation of miR-24 may be responsible for the occurrence of vasospasm in patients with $\mathrm{SAH}$.

Effect of miR-24 on the expression of NOS3. To further confirm whether NOS3 was negatively correlated with miR-24, the mRNA and protein expression levels of NOS3 were analyzed in VSMCs transfected with scramble control, an miR-24 mimic, NOS3 siRNA or an miR-24 inhibitor. As presented in Fig. 5, compared with scramble control, cells transfected with an miR-24 inhibitor exhibited greater expression levels of NOS3 mRNA and protein $(\mathrm{P}<0.05)$. VSMCs transfected with a miR-24 mimic or NOS3 siRNA had reduced expression levels of NOS3 compared with the scramble control $(\mathrm{P}<0.01)$. These observations indicated a negative regulatory association between miR-24 and NOS3.
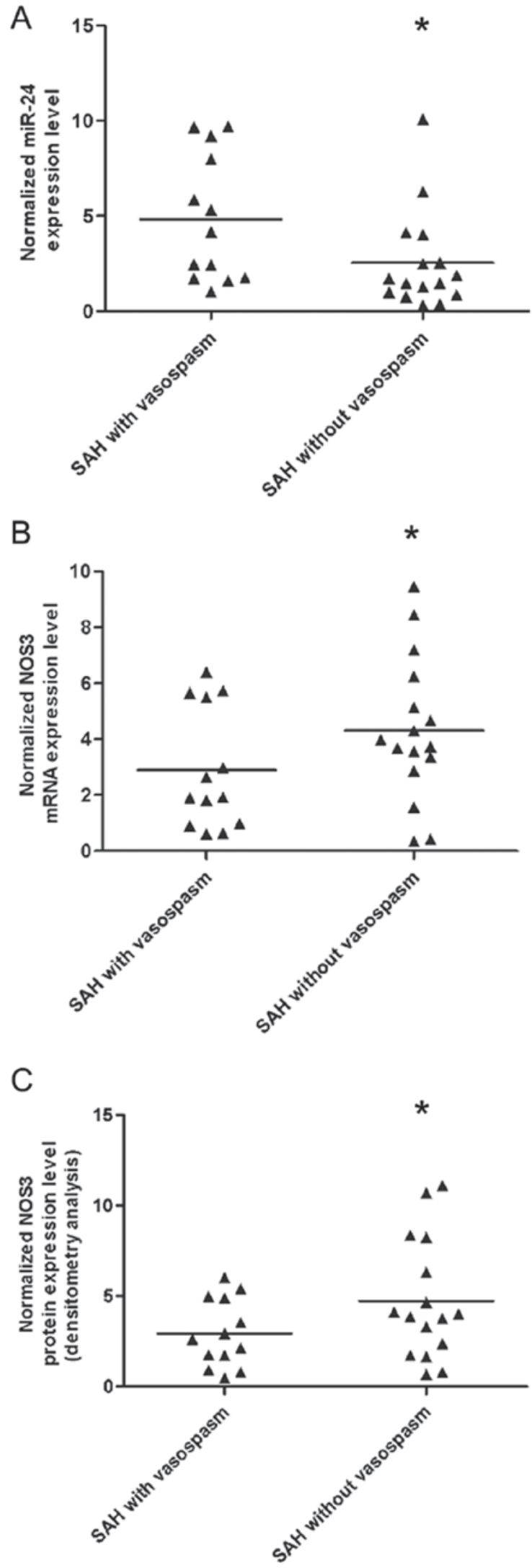

Figure 4. Expression levels of miR-24 and NOS3 in SAH patients with or without vasospasm. (A) Expression levels of miR-24 and (B) mRNA and (C) protein expression levels of NOS3 were analyzed in SAH patients with or without vasospasm. miR-24 expression levels were increased in SAH patients with vasospasm compared with those without, whereas NOS3 expression levels were decreased in SAH patients with vasospasm compared with those without. ${ }^{*} \mathrm{P}<0.05$ vs. SAH with vasospasm. miR, microRNA; NOS3, endothelial nitric oxide synthase; SAH, subarachnoid hemorrhage. 
A
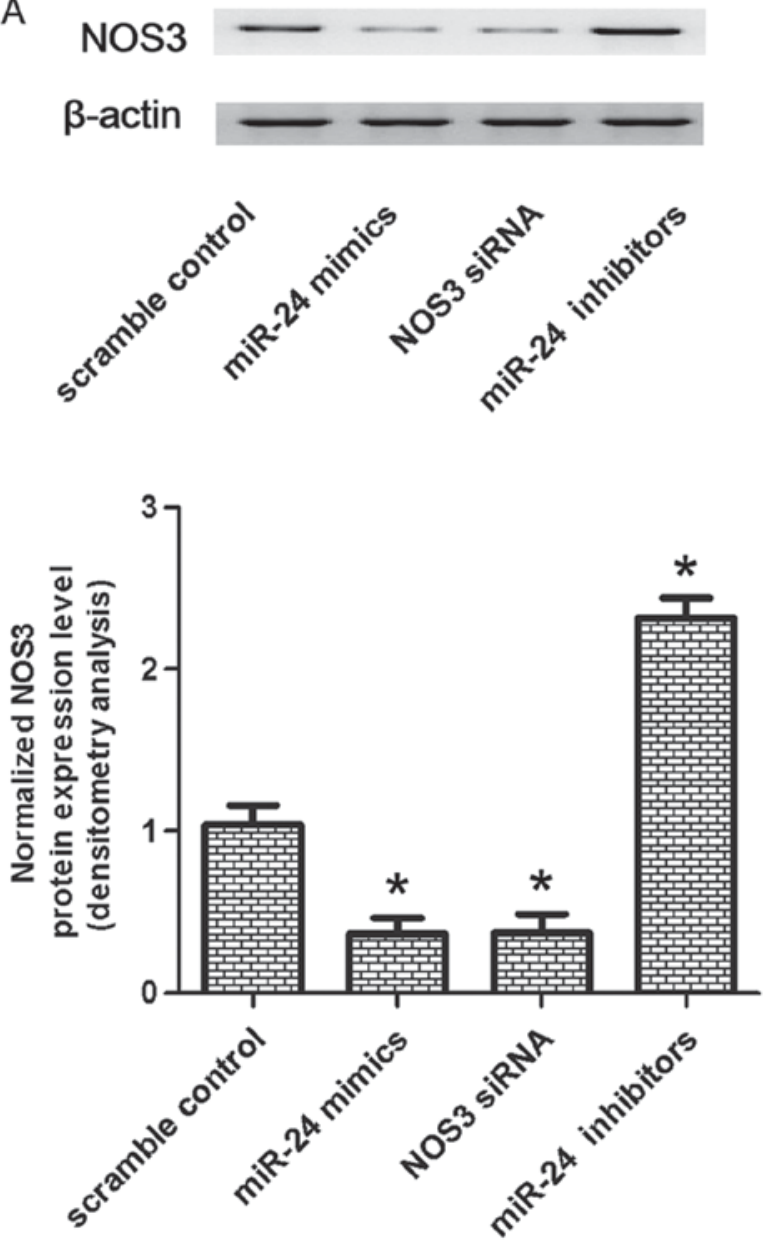

B

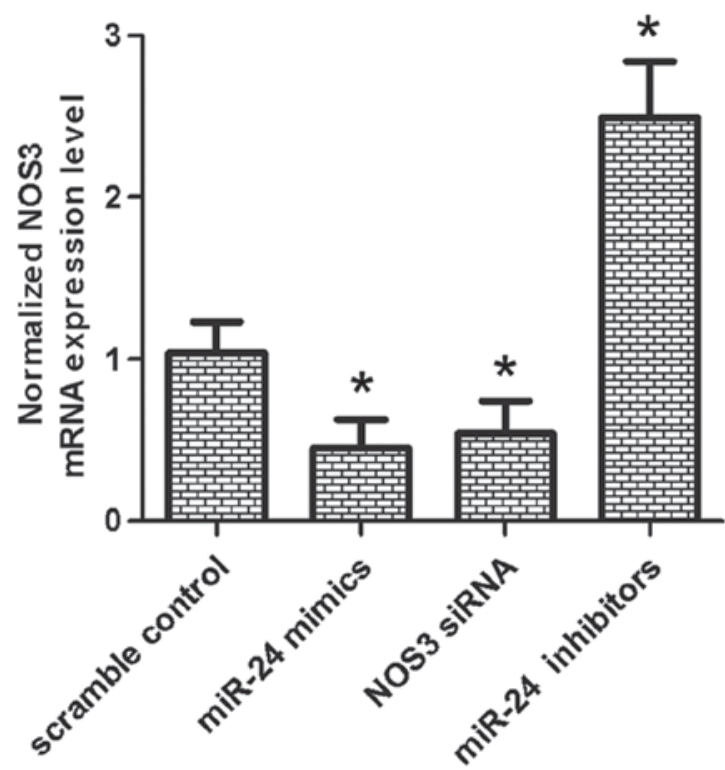

Figure 5. Effect of miR-24 on the expression of NOS3. VSMCs were transfected with an miR-24 mimic, NOS3 siRNA, an miR-24 inhibitor or scramble control, and the NOS3 (A) protein and (B) mRNA expression levels were subsequently assessed by western blotting and reverse transcription-quantitative polymerase chain reaction, respectively. VSMCs transfected with an miR-24 mimic or NOS3 siRNA exhibited decreased expression levels of NOS3 compared with the scramble control, whereas transfection with an miR-24 inhibitor increased NOS3 expression levels. Data are expressed as the mean \pm standard deviation. ${ }^{*} \mathrm{P}<0.05$ vs. scramble control. miR, microRNA; NOS3, endothelial nitric oxide synthase; VSMCs, vascular smooth muscle cells; siRNA, small interfering RNA.

\section{Discussion}

Previous studies have suggested prominent roles for miRNAs in a variety of diseases, including cancer and cardiovascular disease. A growing list of miRNAs, known as 'angiomiR', has been demonstrated to serve cell-independent and cell-dependent regulatory roles in angiogenesis $(25,26)$. The two clusters of miR-23 27 24 include an intergenic miR-23a 27a 24-2 cluster and an intronic miR-23b 27b 24-1 cluster present in the genome of vertebrates (27). Previous studies have demonstrated that in vitro and in vivo angiogenesis via Sprouty2 and Semaphorin $6 \mathrm{~A}$ requires miR-23 and miR-27; miR-24 inhibited angiogenesis through simultaneous regulation of multiple components of the actin cytoskeleton pathways $(28,29)$. A variety of genes downstream of Rho signaling, including LIM domain kinase 2 (Limk2), p21-activating kinase 4 (Pak4) and diaphanous homolog 1 are direct targets of miR-24. Angiogenesis is inhibited by silencing of PAK4 or LIMK2, simulating the phenotype of in vitro miR-24 overexpression, whereas the tube formation defects in miR-24 overexpressing ECs may be partially rescued by overexpression of PAK4 and LIMK2 by adenoviruses (30). Furthermore, subretinal administration of miR-24 inhibits laser-mediated choroidal neovascularization in vivo (30). These results indicated a novel underlying mechanism via which miR-24 regulates angiogenesis and actin cytoskeleton dynamics, and suggested miR-24 may be a potential novel therapeutic agent for the treatment of aberrant angiogenesis via regulation of actin cytoskeleton pathways. The present study performed computational analysis to search for the possible target gene of miR-24, and predicted NOS3 as a target of miR-24 with a potential binding site in the 3'UTR of NOS3. A luciferase assay revealed that miR-24 binds to the NOS3 3'UTR, resulting in a significant decrease in luciferase activity compared with the scramble control. Analysis of tissue samples from SAH patients revealed a negative correlation between expression levels of miR-24 and NOS3 mRNA, confirming the negative regulatory association between miR-24 and NOS3.

A recent study has suggested that $\mathrm{NO}$ acts as an activator of vasomotor tone that may affect development of cerebral vasospasm following SAH (31). NO, as an endogenous vasodilator, is generated by its cleavage from $\mathrm{L}$-arginine by NOS. The generation of NOS, and subsequent NO, is stimulated by gene transcription mediated by numerous intra- and extracellular stimuli. The NO level is reduced following SAH (32). The production of NOS is performed by endothelial cells, neurons and other cells. The reduced level of NO following SAH may be associated with reductions in NO via disruption or scavenging, and/or NOS generation and/or activity (33). Following SAH, the function of NOS3 is damaged in cerebral vessels, which limits vessel relaxation caused by the NOS3-associated elevation in NO generation. Delivery of NOS metabolites and NO donors have demonstrated effectiveness in reducing angiographic cerebral vasospasm $(34,35)$. However, the short half-life of NO, potential toxicity and side effects limit the application of these studies to the clinic $(34,35)$. The present study revealed that miR-24 expression levels increased in SAH patients with vasospasm compared with those without vasospasm, whereas the expression levels of NOS3 decreased in SAH patients with vasospasm compared with those without vasospasm. 
The findings indicated that downregualtion of NOS3 caused by upregulation of miR-24 may be responsible for the occurrence of vasospasm in patients with SAH.

The excessive production of or depletion of the effective vasodilator NO has been investigated with regard to the involvement of secondary complications in the induction of cerebral infarction and ischemia. NO is synthesized non-enzymatically via a range of nitrate-nitrite reduction-oxidation reactions or enzymatically by 3 principal isoforms of NOS including inducible, endothelial and neuronal NOS (35). Physiological levels of NO generation provide a variety of benefits, including preservation of normal vascular tone and vasodilation of the microcirculation, prevention of excessive platelet aggregation and adhesion, antithrombotic effects, VSMC hyperplasia, and suppression of endothelial apoptosis (36). Previous study revealed an association between SAH in mice and oxidative stress in the brain parenchyma, and with NOS3 dysfunction (homodimeric uncoupling) (37). NOS3 uncoupling promoted NO depletion and increased oxidative stress, and was associated with a range of secondary complications including reactive oxygen species release, neuronal apoptosis and microthrombosis (38). The present study investigated the mRNA and protein expression levels of NOS3 in VSMCs transfected with scramble control, an miR-24 mimic, NOS3 siRNA or an miR-24 inhibitor. Transfection with an miR-24 inhibitor increased the expression levels of NOS3, whereas transfection with an miR-24 mimic or NOS3 siRNA decreased NOS3 expression levels. These observations indicated that there was a negative regulatory association between miR-24 and NOS3.

In conclusion, the results of the present study demonstrated that miR-24 directly targets NOS3, and downregulation of NOS3 may induce vasospasm following SAH. This may be caused by the upregualtion of miR-24 in VSMCs. The present study indicated that miR-24 is a potential therapeutic target for the treatment of SAH/vasospasm.

\section{Acknowledgements}

Not applicable.

\section{Funding}

No funding was received.

\section{Availability of data and materials}

The datasets used and/or analyzed during the current study are available from the corresponding author on reasonable request.

\section{Authors' contributions}

HTL and JW were responsible for study planning, data collection, data analysis and interpretation, preparation of the manuscript and literature analysis. SFL performed data collection, data analysis and interpretation. LC performed data analysis and interpretation and preparation of the manuscript. WZT was responsible for data analysis and interpretation and literature analysis; and YGF for data collection, data analysis and interpretation.

\section{Ethics approval and consent to participate}

The present study was approved by the Human Ethics Committee of Qingdao University (Qingdao, China). Participants or their first-degree relatives signed informed consent forms prior to samples being obtained.

\section{Consent for publication}

Not applicable.

\section{Competing interests}

The authors declare that they have no competing interests.

\section{References}

1. Connolly ES Jr, Rabinstein AA, Carhuapoma JR, Derdeyn CP, Dion J, Higashida RT, Hoh BL, Kirkness CJ, Naidech AM, Ogilvy CS, et al: Guidelines for the management of aneurysmal subarachnoid hemorrhage: A guideline for healthcare professionals from the American Heart Association/american Stroke Association. Stroke 43: 1711-1737, 2012.

2. Suarez JI, Tarr RW and Selman WR: Aneurysmal subarachnoid hemorrhage. N Engl J Med 354: 387-396, 2006.

3. Sarrafzadeh A, Haux D, Sakowitz O, Benndorf G, Herzog H, Kuechler I and Unterberg A: Acute focal neurological deficits in aneurysmal subarachnoid hemorrhage: Relation of clinical course, CT findings, and metabolite abnormalities monitored with bedside microdialysis. Stroke 34: 1382-1388, 2003.

4. Quyyumi AA, Dakak N, Andrews NP, Gilligan DM, Panza JA and Cannon RO III: Contribution of nitric oxide to metabolic coronary vasodilation in the human heart. Circulation 92: 320-326, 1995.

5. Weir B and MacDonald L: Cerebral vasospasm. Clin Neurosurg 40: 40-55, 1993.

6. Khurana VG, Smith LA, Baker TA, Eguchi D, O'Brien T and Katusic ZS: Protective vasomotor effects of in vivo recombinant endothelial nitric oxide synthase gene expression in a canine model of cerebral vasospasm. Stroke 33: 782-789, 2002.

7. Woszczyk A, Deinsberger W and Böker DK: Nitric oxide metabolites in cisternal CSF correlate with cerebral vasospasm in patients with a subarachnoid haemorrhage. Acta Neurochir (Wien) 145: 257-263; discussion 263-644, 2003.

8. Sadamitsu D, Kuroda Y, Nagamitsu T, Tsuruta R, Inoue T, Ueda T, Nakashima K, Ito $\mathrm{H}$ and Maekawa T: Cerebrospinal fluid and plasma concentrations of nitric oxide metabolites in postoperative patients with subarachnoid hemorrhage. Crit Care Med 29: 77-79, 2001.

9. Khurana VG, Smith LA, Weiler DA, Springett MJ, Parisi JE, Meyer FB, Marsh WR, O'Brien T and Katusic ZS: Adenovirus-mediated gene transfer to human cerebral arteries. J Cereb Blood Flow Metab 20: 1360-1371, 2000.

10. Moncada S, Palmer RM and Higgs EA: Nitric oxide: Physiology, pathophysiology, and pharmacology. Pharmacol Rev 43: 109-142, 1991.

11. Dumont AS, Dumont RJ, Chow MM, Lin CL, Calisaneller T, Ley KF, Kassell NF and Lee KS: Cerebral vasospasm after subarachnoid hemorrhage: Putative role of inflammation. Neurosurgery 53: 123-133; discussion 133-135, 2003.

12. Khurana VG, Sohni YR, Mangrum WI, McClelland RL, O'Kane DJ, Meyer FB and Meissner I: Endothelial nitric oxide synthase gene polymorphisms predict susceptibility to aneurysmal subarachnoid hemorrhage and cerebral vasospasm. J Cereb Blood Flow Metab 24: 291-297, 2004.

13. Yoshimura M,Nakayama M, Shimasaki Y, Ogawa H, Kugiyama K, Nakamura S, Ito T, Mizuno Y, Harada E, Yasue H, et al: A T-786- $>$ C mutation in the 5'-flanking region of the endothelial nitric oxide synthase gene and coronary arterial vasomotility. Am J Cardiol 85: 710-714, 2000.

14. Saugstad JA: MicroRNAs as effectors of brain function with roles in ischemia and injury, neuroprotection, and neurodegeneration. J Cereb Blood Flow Metab 30: 1564-1576, 2010. 
15. Cordes KR, Sheehy NT, White MP, Berry EC, Morton SU, Muth AN, Lee TH, Miano JM, Ivey KN and Srivastava D: miR-145 and miR-143 regulate smooth muscle cell fate and plasticity. Nature 460: 705-710, 2009.

16. Vikman P, Ansar S, Henriksson M, Stenman E and Edvinsson L: Cerebral ischemia induces transcription of inflammatory and extracellular-matrix-related genes in rat cerebral arteries. Exp Brain Res 183: 499-510, 2007.

17. Vikman P, Beg S, Khurana TS, Hansen-Schwartz J and Edvinsson L: Gene expression and molecular changes in cerebral arteries following subarachnoid hemorrhage in the rat. J Neurosurg 105: 438-444, 2006.

18. Johansson S, Povlsen GK and Edvinsson L: Expressional changes in cerebrovascular receptors after experimental transient forebrain ischemia. PLoS One 7: e41852, 2012.

19. Edvinsson LI and Povlsen GK: Vascular plasticity in cerebrovascular disorders. J Cereb Blood Flow Metab 31: 1554-1571, 2011.

20. Liu D, Han L, Wu X, Yang X, Zhang Q and Jiang F: Genome-wide microRNA changes in human intracranial aneurysms. BMC Neurol 14: 188, 2014.

21. Starke RM, Kim GH, Komotar RJ, Hickman ZL, Black EM, Rosales MB, Kellner CP, Hahn DK, Otten ML, Edwards J, et al: Endothelial nitric oxide synthase gene single-nucleotide polymorphism predicts cerebral vasospasm after aneurysmal subarachnoid hemorrhage. J Cereb Blood Flow Metab 28: 1204-1211, 2008

22. Khurana VG, Sohni YR, Mangrum WI, McClelland RL, O'Kane DJ, Meyer FB and Meissner I: Section on cerebrovascular surgery: Galbraith Award: Endothelial nitric oxide synthase (eNOS) and heme oxygenase-1 (HO-1) gene polymorphisms predict susceptibility to aneurysmal subarachnoid hemorrhage (SAH) and post-SAH cerebral vasospasm. Clin Neurosurg 51: 343-350, 2004

23. Livak KJ and Schmittgen TD: Analysis of relative gene expression data using real-time quantitative PCR and the 2(-Delta Delta C(T)) method. Methods 25: 402-408, 2001

24. Lang B, Shang C and Meng L: Targeted Silencing of S100A8 Gene by miR-24 to Increase Chemotherapy Sensitivity of Endometrial Carcinoma Cells to Paclitaxel. Med Sci Monit 22: 1953-1958, 2016.

25. Small EM and Olson EN: Pervasive roles of microRNAs in cardiovascular biology. Nature 469: 336-342, 2011.

26. Garofalo $M$ and Croce CM: microRNAs: Master regulators as potential therapeutics in cancer. Annu Rev Pharmacol Toxicol 51: $25-43,2011$.
27. Wang $\mathrm{S}$ and Olson EN: AngiomiRs-key regulators of angiogenesis. Curr Opin Genet Dev 19: 205-211, 2009.

28. Zhou Q, Gallagher R, Ufret-Vincenty R, Li X, Olson EN and Wang S: Regulation of angiogenesis and choroidal neovascularization by members of microRNA-23 27 24 clusters. Proc Natl Acad Sci USA 108: 8287-8292, 2011.

29. Urbich C, Kaluza D, Frömel T, Knau A, Bennewitz K, Boon RA, Bonauer A, Doebele C, Boeckel JN, Hergenreider E, et al: MicroRNA-27a/b controls endothelial cell repulsion and angiogenesis by targeting semaphorin 6A. Blood 119: 1607-1616, 2012

30. Zhou Q, Anderson C, Zhang H, Li X, Inglis F, Jayagopal A and Wang S: Repression of choroidal neovascularization through actin cytoskeleton pathways by microRNA-24. Mol Ther 22: 378-389, 2014.

31. Siuta M, Zuckerman SL and Mocco J: Nitric oxide in cerebral vasospasm: Theories, measurement, and treatment. Neurol Res Int 2013: 972417, 2013.

32. Jung CS, Iuliano BA, Harvey-White J, Espey MG, Oldfield EH and Pluta RM: Association between cerebrospinal fluid levels of asymmetric dimethyl-L-arginine, an endogenous inhibitor of endothelial nitric oxide synthase, and cerebral vasospasm in a primate model of subarachnoid hemorrhage. J Neurosurg 101: 836-842, 2004

33. Kim P, Schini VB, Sundt TM Jr and Vanhoutte PM: Reduced production of cGMP underlies the loss of endothelium-dependent relaxations in the canine basilar artery after subarachnoid hemorrhage. Circ Res 70: 248-256, 1992.

34. Hino A, Tokuyama Y, Weir B, Takeda J, Yano H, Bell GI and Macdonald RL: Changes in endothelial nitric oxide synthase mRNA during vasospasm after subarachnoid hemorrhage in monkeys. Neurosurgery 39: 562-567; discussion 567-568, 1996.

35. Afshar JK, Pluta RM, Boock RJ, Thompson BG and Oldfield EH: Effect of intracarotid nitric oxide on primate cerebral vasospasm after subarachnoid hemorrhage. J Neurosurg 83: 118-122, 1995.

36. van Faassen EE, Bahrami S, Feelisch M, Hogg N, Kelm M, Kim-Shapiro DB, Kozlov AV, Li H, Lundberg JO, Mason R, et al: Nitrite as regulator of hypoxic signaling in mammalian physiology. Med Res Rev 29: 683-741, 2009.

37. Förstermann U and Münzel T: Endothelial nitric oxide synthase in vascular disease: From marvel to menace. Circulation 113: 1708-1714, 2006.

38. Sabri M, Ai J, Knight B, Tariq A, Jeon H, Shang X, Marsden PA and Loch Macdonald R: Uncoupling of endothelial nitric oxide synthase after experimental subarachnoid hemorrhage. J Cereb Blood Flow Metab 31: 190-199, 2011. 